SECCIÓN ABIERTA 

REVISTA DE DERECHO UNED, NÚM. 9, 2011

\title{
MANUEL J. GARCÍA GARRIDO E ALESSANDRO CORBINO DOTTORI HONORIS CAUSA A MOSCA ${ }^{1}$
}

\author{
FEDERICO FERNÁNDEZ DE BUJÁN
}

\section{PREMESSA}

Con grato ricordo assumo il compito di compilare una Cronaca della cerimonia di nomina a Dottori Honoris Causa dell'Università Statale Accademica degli Studi di Scienze Umanistiche di Mosca dei Professori Manuel J. García Garrido (UNED, Madrid) e Alessandro Corbino (Università di Catania). Questa Istituzione universitaria dell' Accademia delle Scienze di Russia vanta le Facoltà di Storia e di Diritto di maggiore rilevanza scientifica e di più vasta reputazione nel mondo forense della Russia.

La solenne cerimonia del conferimento dei Diplomi di «Doctor honoris causa» si è tenuta nell'ambito del VII Seminario internazionale scientifico «Diritto romano e attualità», svoltosi sul tema "La terminologia giuridica nel Diritto Romano e odierno: la decisione giudiziaria e sua esecuzione», organizzato in collaborazione fra il Centro di Studi di Diritto Romano di Mosca², l'Università Karlova di Praga e l'Accademia Giuridica del Ministero della Giustizia della Federazione Russa.

Scrivo questa Cronaca con l'intenzione di fornire al lettore una sintesi fedele ed essenziale delle parole pronunciate nella solenne occasione e renderlo così consapevole dell'andamento della Cerimo-

${ }^{1}$ La presente reproduce una Crónica publicada en distintas Revistas italianas iusprivatistas y romanistas.

2 Questo Centro è fondato dall'Istituto di Storia Universale dell'Accademia delle Scienze di Russia e dalla Facoltà di Giurisprudenza dell'Università degli Studi di Mosca «M.V.Lomonosov». 
nia. Per questa ragione lo stile espositivo sarà quello della riproduzione fedele, quasi stenografica, dell'intensa giornata moscovita.

L'alto riconoscimento accademico accordato si aggiunge ai tanti riconoscimenti che sono stati tributati, nell'ambito delle loro feconde traiettorie umane, scientifiche ed accademiche, a due romanisti e giuristi che rappresentano, nei rispettivi Paesi, e nel panorama giuridico internazionale, la prova irrefutabile dell'eccellenza scientifica nello studio del Diritto Romano e la sua perenne attualità e validità nel mondo del diritto.

Inoltre, a mio avviso, il Dottorato Honoris Causa attribuito ai professori Corbino e García Garrido nella stessa occasione ha un alto valore simbolico e può rappresentare, in certa misura, una prova dell'unione cordiale esistente tra l'Italia e la Spagna, testimoniata in un'intera vita di collaborazione scientifica e di amicizia fra i due studiosi onorati a Mosca.

\section{RESOCONTO DELLA CERIMONIA}

Presiede la Prof.ssa Letizia Vacca, insigne giurista italiana, alla presenza del Magnifico Rettore dell'Accademia di Diritto del Ministero della Giustizia della Federazione Russa, Prof. Serghej Gerasimov. Ha inizio la cerimonia e la Prof.ssa Vacca dichiara:

Nell'apertura di questa seduta abbiamo la cerimonia molto importante del conferimento del titolo di Doctor Honoris Causa a due Colleghi non solo di grande capacità scientifica, ma anche di grande umanità e di grande simpatia e ai quali mi lega un'antica amicizia. Sono quindi particolarmente felice di assistere e partecipare a questa cerimonia. Successivamente aggiunge: Do subito la parola al Rettore Michail Bibikov dell'Università dell'Accademia delle Scienze di Russia per introdurre questa cerimonia.

Prende la parola il Rettore dell'Università, insigne filologo e storico, che sottolinea l'importanza che questo evento riveste per la sua Istituzione. Nel suo breve intervento esprime con soddisfazione la gratitudine che l'Accademia delle Scienze di Russia, e specialmente l'Università Statale Accademica degli Studi di Scienze Umanistiche, esprime ai nuovi Dottori che entrano a far parte del suo Consiglio accademico, per il prezioso ed importante contributo da loro svolto ai fini della sua promozione e rilevanza internazionale. Il Rettore Bibikov mette inoltre in risalto il significato del riconoscimento universitario e scientifico e le valutazioni che l'organo di governo dell'Uni- 
versità ha tenuto in considerazione ai fini del conferimento del massimo onore accademico del dottorato honoris causa.

Di seguito prende la parola il illustre e caro collega Prof. Leonid Kofanov, che tanto bene ha fatto per lo studio ed lo sviluppo della ricerca del Diritto Romano nella Russia. E fà la Laudatio di rito, affermando: Parlerò russo per rendere possibile una chiara comprensione del significato della cerimonia per i nostri Colleghi russi.

Con tono disteso ed affettuoso, ma non senza solennità, si riferisce inizialmente al Prof. García Garrido, di cui mette in rilievo, sotto diversi aspetti, gli altissimi meriti giuridici, universitari e politici:

Manuel Jesus Garcia Garrido è nato 28 aprile 1928 in Spagna e la sua vita è stata piena e ricca di contenuti. È stato Preside della Facoltà di Giurisprudenza e successivamente Rettore dell'Università di Compostela; successivamente è stato fondatore e primo Rettore dell'Università nazionale dell'educazione a distanza (UNED) della Spagna, che adesso è molto famosa in tutto il mondo ed ha filiali non solo in Europa, ma anche in quasi tutti i paesi dell'America Latina. Nel 1978 Egli è stato eletto nel Parlamento della Spagna. Si può dire che è stato uno degli autori della Costituzione vigente della Spagna.

Si sofferma quindi sulla sua straordinaria personalità scientifica:

Tuttavia, lo scopo e il senso di tutta la sua vita è stato sempre, e resta anche adesso, l'attività scientifica nel campo giusromanistico. Egli è l'autore di centinaia di pubblicazioni, di decine di monografie sul Diritto Romano; è uno dei traduttori del Digesto di Giustiniano nella lingua spagnola. Basta dire che proprio grazie al suo lavoro titanico oggi qualsiasi biblioteca di diritto romano ha l'edizione in molti volumi delle Digestorum Similitudines ${ }^{3}$.

Sottolinea anche l'attiva e generosa partecipazione del Prof. García Garrido con la sua Università, quando ne ricorda l'aiuto prestato nei primi anni e l'assidua partecipazione alla regolare attività di docenza:

... collabora sempre molto attivamente con le sue lezioni al Corso di perfezionamento in diritto romano del Centro di studi di diritto romano a Mosca... e coll'Università Accademica degli Studi di Scienze Umanistiche.

Il professor Kofanov esprime quindi la propria gratitudine all'Onorato per aver concesso l'autorizzazione affinché il suo famoso manuale «Diritto romano privato: casi, azioni, istituzioni» ${ }^{4}$ venisse tradotto in lingua russa ad opera di M.V. Lomonosov:

3 García Garrido M., Reinoso-Barbero F. Digestorum Similitudines. Vol. I-XI. Madrid, 1994.

${ }_{4}$ Гарсиа Гарридо М.Е. Римское частное право. Казусы, иски, институции. Перевод с испанского. Отв. ред. Л.Л.Кофанов- М.: Статут, 2005. -812 с. 
Questo manuale in un certo senso ha un carattere enciclopedico, e secondo l'opinione degli studiosi, dei professori e degli studenti di Russia è diventato uno dei migliori manuali di diritto romano sia per gli studenti, sia per $i$ docenti.

Conclude dichiarando solennemente:

Secondo la raccomandazione del suo allievo, Prof. Federico Fernández de Buján, il Rettore ha portato alla discussione la domanda sulla consegna del titolo Doctor Honoris Causa e il 13 settembre 2011 il Consiglio scientifico dell'Università ha votato unanimemente la decisione in favore della consegna del titolo al Prof. Manuel García Garrido.

[In questo momento ha luogo la cerimonia di consegna solenne del Diploma di cui, per ragioni di ordine testuale, riferirò in seguito.]

Al termine il Rettore dichiara:

Adesso la presentazione del prof. Alessandro Corbino.

Anche questa viene affidata al Prof. Leonid Kofanov, che la pronuncia con grande emozione:

Permettetemi di presentare il prof. Alessandro Corbino. Io sono molto emozionato poiché mi considero Suo allievo. È nato 19 agosto 1945 a Roma, ed è professore della Facoltà di Giurisprudenza dell'Università di Catania. Durante la sua ricca vita scientifica ed accademica è stato Preside della Facoltà di Giurisprudenza dell'Università di Catanzaro. Negli anni 2003-2007 è stato Difensore Civico del Comune di Catania. Dal 2000 è componente del Comitato Nazionale di Valutazione del Sistema Universitario. Dal 2010 è componente laico del Consiglio di Giustizia Amministrativa della Regione Siciliana.

Ne sottolinea quindi, con nitida chiarezza, l'intensa ed efficace collaborazione con le Istituzioni russe che gli rendono omaggio con il conferimento del dottorato honoris causa:

Da più di 20 anni è il Presidente del Centro romanistico internazionale "Copanello", che unisce $i$ romanisti della maggior parte dei paesi d'Europa, dell'America latina e dell'Asia. Quest'anno si è compiuto il giubileo di 20 anni della sua collaborazione con l'Istituto della storia universale dell'Accademia delle scienze di Russia (..) alla fine del 2005 sulla base del programma ERASMUS, Egli ha creato - e dagli ultimi anni presieduto-il Corso internazionale interuniversitario «Ius civile dell'Europa comune» del consorzio delle Università europee sotto la direzione dell'Università di Catania. In questo consorzio, insieme con le Università d'Italia, di Spagna, di Ungheria et cet. è inclusa anche l'Università Accademica degli Studi di Scienze Umanistiche dell'Accademia delle Scienze di Russia.

Ne esprime quindi l'eccelsa personalità scientifica: 
È uno dei più eminenti scienziati europei, è l'autore di centinaia di articoli e di decine delle monografie e manuali sul diritto romano. L'edizione del manoscritto dei Digesta di Giustiniano Littera Florentina (quella che è riconosciuta dai giuristi come "Bibbia dei giuristi«) è la più famosa in tutto il mondo. In collaborazione col prof. Dmitrij Dodzdev della Facoltà di Giurisprudenza della nostra Università, Egli è stato iniziatore della traduzione e della pubblicazione in Russia del manuale del diritto romano del suo Maestro, Professore Cesare Sanfilippo ${ }^{5}$.

$\mathrm{Al}$ termine di ciascuna delle presentazioni che ho riportato, rivestite dalla forma della Laudatio, si è quindi proceduto alla solenne nomina dei due Dottori Honoris Causa. Essendovi assoluta identità nella formula del conferimento del Diploma e nel cerimoniale, li riporto in formulazione comune, con il nome di entrambi.

Il Rettore, Prof. Michail Bibikov, dichiara:

Sono felice di dare lettura della diploma, redatto in latino:

CONSILIVM DOCTORVM PVBLICAE VNIVERSITATIS ACADEMICAE HVMANITATVM aestimationis causa meritorum magnorum in campo artis, eruditionis, humanitatis et iuris gentium viro clarissimo professori MANUEL JESUS GARCIA GARRIDO/ ALESSANDRO CORBINO HONORIS CAVSA DOCTORIS (...) nomen tribuit.

Il Rettore invita il Prof. Manuel J. Garcia Garrido/il Prof. Alessandro Corbino ad avvicinarsi e conferisce loro la decorazione. Consegna loro il diploma di Dottore honoris causa e la statuetta di bronzo dell'antica divinità greca Nike. La Prof.ssa Sofia Safronova, del Centro di studi di diritto romano, dell'Accademia di Giustizia della Corte suprema della Federazione Russa, fa loro dono di un mazzo di fiori. Al termine di questo momento protocollare, la professoressa Letizia Vacca concede la parola al professore García Garrido.

L'Onorato dà inizio al proprio intervento, notevolmente emozionato, ringraziando il Rettore e l'organo di governo dell'Università. Porge i propri ringraziamenti anche al Prof. Leonid Kofanov per i tanti momenti felici vissuti in occasione di congressi, seminari e corsi in Italia, Spagna e Russia, nonché per le sentite parole che questi gli ha rivolto. Dichiara di sentirsi felice per aver potuto conoscere, nei diversi viaggi effettuati, la realtà sociale ed accademica russa, realizzando così un sogno della sua giovinezza.

In questo senso afferma:

5 Санфилиппо Чезаре. Курс римского частного права: учебник / Под ред. Д.В. Дождева.М.: Издательство БЕК, 2000 (и последующие переиздания). 
La mia riconoscenza deve anche estendersi alla possibilità di conoscere la Russia, nei viaggi in cui ho potuto apprezzare le meraviglie di quello che é un grande continente più che una Nazione, e vivere la Storia e entrare in luoghi che sempre ho sognato di conoscere. L'arte bizantina delle Chiese e dei Monasteri, la maestosità delle edifici, nel Yaroslasvl, le silenziose strade e le verdi terre bagnate dal Volga, percorsi con l'amico scomparso Gennaro Franciosi, anche le Pagine gloriose della letteratura, leggende di eroi, amori e tragedia di Puskin, Dostoevskij, Tolstoj e Pasternak, e le musiche e melodie di Chaikovskij che risuonano sempre.

Il professor García Garrido dichiara quindi il suo impegno a vita al servizio dell'Università:

Se il libro di Giobbe afferma: "L'uomo sta in questa terra per adempiere un servizio", nella mia vita lo spirito di servizio alla Università, agli allievi, agli studenti sempre ha presieduto le mie opere.

Esprime la sua grande felicità per poter condividere questa cerimonia con Alessandro Corbino:

Sono molto felice di poter comparticipare in questa cerimonia con il carissimo amico Alessandro Corbino, al quale mi sento unito dopo $i$ molti anni di stretti rapporti. La sua direzione, che comparte con Metro, ha elevato il Centro Copanello ai massimi livelli della Istituzione di maggiore importanza e diffusione del Diritto Romano nel mondo.

E conclude:

Devo finire tornando alla gratitudine. A Lourdes, mia moglie che tanto mi aiuta, agli affettuosi allievi Fernando Reinoso e Federico de Bujan, e agli allievi e amici che sono presenti (...) e aggiunge: Ringrazio con tutto il mio cuore per la generosa concessione del Dottorato Honoris causa (...) beneficio che avrò presente sforzandomi di essere degno della cooptazione al suo prestigioso Collegio di Professori e saggi Dottori. Come nel canto medievale spero che l'Universitá «vivat, crescat et floreat». Finisce recitando, in italiano, una bella poesia ben conosciuta in russo, di Afanasio Fet.

La Prof.ssa Letizia Vacca invita quindi a parlare il Prof. Corbino, che inizia affermando:

È con profonda emozione che prendo la parola in questa occasione. L'onore che ricevo è grandissimo. Certamente al di sopra di ogni mio merito. So di doverlo alla benevolenza di Leonid Kofanov (...) ho anche la fortuna di condividere questa gioia con un Amico carissimo, il prof. Manuel Jesús García Garrido (...) Il Centro Copanello lo annovera da sempre tra i componenti più autorevoli del proprio comitato scientifico internazionale. 
Passa quindi a fare memoria storica del Centro, che nacque a Catanzaro nel 1984, e del suo sviluppo:

... nessuno - e meno di tutti io- avrebbe potuto prevedere gli sviluppi che ne sarebbero venuti: $i$ convegni di Copanello (ne celebreremo a giugno prossimo la XVI edizione); la riproduzione fotografica del codice Laurenziano delle Pandette di Giustiniano; i seminari di Soverato (di cui abbiamo appena realizzato la XI edizione) e via via le molte altre cose che ne sono seguite tra le quali mi piace qui ricordare il corso di laurea internazionale in «Ius civile dell'Europa comune».

Quindi, con grande partecipazione, dichiara:

Fare programmi a 40 anni non è lo stesso che farli a quasi settanta. Ma se posso osare di farne ancora non è perché io abbia perso il senso del tempo (e dell'inesorabile accorciarsi di quello che mi può ancora essere concesso). Ė perché il lavoro fatto è stato un lavoro corale. Ed esso perciò prosegue ormai-sicuro e solido-per il continuo aggiungersi, ai vecchi, di nuovi e più giovani compagni di lavoro.

E, con contenuta ma evidente vibrazione, parla di Soverato. Quel Seminario che io ho qualificato, in una delle sue Giornate, come stella polare nella formazione delle nuove generazioni di romanisti. Così, grazie alla sua iniziativa, tutti abbiamo potuto godere di un'amabile occasione intellettuale e personale. Il Seminario ha un'atmosfera cordiale che ha fatto di Soverato uno splendido pretesto per l'incontro umano. Ritengo che solo il magistero, rivestito di auctoritas, dei Professori Alessandro Corbino e Antonino Metro, oggi proseguito dall'impegno della loro allieva, professoressa Piro, abbiano potuto fare sì che Soverato rappresenti un appuntamento atteso con trepidazione dai suoi partecipanti, superando ogni ostacolo che ne rendeva difficile la realizzazione. Ancora Corbino:

Da quei seminari-cominciati venti anni fa, nel 1991-è nata una comunità nella comunità (...) si è creata da subito un'atmosfera speciale. Mosca, Praga, Sofia, Santiago del Cile, Buenos Aires, S. Paolo, Shangai, Ankara, Istanbul, Budapest e le tante altre sedi sono divenute, ogni biennio -con Madrid, Parigi, Köln, Amsterdam, Varsavia, Roma, Napoli, Torino, Firenze, Messina, Catania, Catanzaro (e potrei a lungo continuare)-, "quartieri», regiones di un'unica civitas (...) Leonid Kofanov, Michail Screjpek, Konstantin Tanev, Sara Correa, Patricio Carvajal, Federico Fernández de Buján, Adriana Garcia Netto, Liong Zhang, Isa Piro, Francesco Milazzo (ma l'elenco è solo esemplificativo e per l'Italia assolutamente circoscritto alle sole persone, per così dire, "di casa«) hanno realizzato non solo una solidissima personale e reciproca amicizia, ma hanno anche vissuto la loro presenza al seminario come sostenuta da una ragione unica, assolutamente solidale. 
Al termine del turno di interventi, la Prof.ssa Letizia Vacca chiude solennemente la cerimonia:

Vorrei esprimere ancora una volta il piacere di aver partecipato a questa cerimonia e un augurio personale al professor García Garrido e al professor Corbino, che hanno anche nelle loro parole di ringraziamento dimostrato il loro alto livello umano e scientifico e ancora una volta testimoniato gli elementi di una fraternità all'interno della comunità internazionale, che non può che arricchire non solo il diritto romano, ma tutta la scienza giuridica e anche noi sul piano umano e personale.

\section{POSTFATIO}

In un'altra occasione ho scritto: "Beati i discepoli che hanno autentici Maestri» e "Beati i Maestri che hanno buoni discepoli» ${ }^{6}$. Né tutti gli studenti all'alba del loro percorso hanno potuto avere un Maestro, né tutti gli universitari di lungo corso hanno potuto avuto discepoli. In questo senso i Maestri García Garrido e Corbino sono stati beati, così come lo siamo noi, che abbiamo avuto il privilegio di formarci accanto a loro. Così, parafrasando Virgilio ${ }^{7}$ :

Per dove passarono i Maestri García Garrido e Corbino cresce ancora l'erba che loro seminarono.

Inoltre entrambi hanno saputo ricoprire non solo la figura del Professore, nel rapporto con i rispettivi allievi, ma la loro qualità accademica ha saputo coniugarsi con un aspetto umano amabile, cortese e soprattutto affettuoso. García Garrido e Corbino -idem animus, eadem voluta- hanno saputo e voluto infondere nei loro discepoli, così come in tutti coloro che si sono avvicinati a loro, la passione per la scienza romanistica.

Sono pertanto riusciti, e continuano ancor oggi a fare in modo che le generazioni più giovani possano formarsi con il rigore richiesto dal nostro compito intellettuale. Hanno tradotto in realtà il bel pensiero di Saint-Exupery:

Se vuoi costruire una barca, non iniziare a cercare il legno o distribuendo il lavoro, ma per prima cosa riunisci un gruppo di uomini e instilla in loro la nostalgia di solcare il mare appassionante ed infinito ${ }^{8}$.

${ }^{6}$ Fernández de Buján, F., Aproximación al Profesor Manuel J. García Garrido, en prólogo de Miscelánea Romanística, vol. I, servicio de publicaciones UNED. Madrid, p. 29.

7 Eneide, VI, 851,3.

8 Cittadella, sezione LXXV. 
E declama Dante, dalla grandezza del suo messaggio e linguaggio:

O voi ch'avete li 'ntelletti sani, mirate la dottrina che s'asconde sotto "l velame de li versi strani"

Questi due grandi Maestri hanno esercitato l'«intelletto sano»di cui parla il poeta. Hanno incarnato il perfetto compimento del munus docendi con i loro alunni e discepoli. Per mezzo del loro luminoso magistero abbiamo potuto scoprire gli arcani che tante volte si nascondono sotto al velo dei frammenti giurisprudenziali. Hanno insegnato con vastità di dottrina, genialità di intelletto, fede incorruttibile nella grandezza del Diritto Romano, così come, con la mentalità del iuvat qui iubet, affidando un compito intellettuale, ci hanno aiutati, con il loro tempo e sapere, a realizzare quanto affidatoci.

La dottrina romanista gioisce per il riconoscimento internazionale a questi illustri e riconosciuti Maestri, ai quali auguriamo di ricevere rinnovati e solenni onori. Noi tutti, come loro discepoli, abbiamo nel cuore una grande gioia per questo nuovo riconoscimento delle persone che stimiamo ed ammiriamo. Concludo ringraziando il caro amico Salvo Randazzo che, con sentimenti di affetto devoto nei confronti del suo Maestro professore Corbino e di pari ammirazione nei confronti del professore García Garido mi ha affidato il compito di redigere questa Cronaca.

${ }^{9}$ Divina Commedia, Inferno, canto IX, 63. 
